Article

\title{
New Time-Varying Sliding Surface for Switching Type Quasi-Sliding Mode Control
}

\author{
Katarzyna Adamiak *(D) and Andrzej Bartoszewicz (D) \\ Institute of Automatic Control, Łódź University of Technology, 18/22 Bohdana Stefanowskiego St., \\ 90-924 Łódź, Poland; andrzej.bartoszewicz@p.lodz.pl \\ * Correspondence: katarzyna.adamiak@p.lodz.pl
}

Citation: Adamiak, K.; Bartoszewicz, A. New Time-Varying Sliding Surface for Switching Type Quasi-Sliding Mode Control. Energies 2021, 14, 3811. https://doi.org/10.3390/en14133811

Academic Editors: Mojtaba

Ahmadieh Khanesar and

Amin Hajizadeh

Received: 27 May 2021

Accepted: 17 June 2021

Published: 24 June 2021

Publisher's Note: MDPI stays neutral with regard to jurisdictional claims in published maps and institutional affiliations.

Copyright: (c) 2021 by the authors. Licensee MDPI, Basel, Switzerland. This article is an open access article distributed under the terms and conditions of the Creative Commons Attribution (CC BY) license (https:// creativecommons.org/licenses/by/ $4.0 /)$.

\begin{abstract}
This study considers the problem of energetical efficiency in switching type sliding mode control of discrete-time systems. The aim of this work is to reduce the quasi-sliding mode band-width and, as follows, the necessary control input, through an application of a new type of time-varying sliding hyperplane in quasi-sliding mode control of sampled time systems. Although time-varying sliding hyperplanes are well known to provide insensitivity to matched external disturbances and uncertainties of the model in the whole range of motion for continuous-time systems, their application in the discrete-time case has never been studied in detail. Therefore, this paper proposes a sliding surface, which crosses the system's representative point at the initial step and then shifts in the state space according to the pre-generated demand profile of the sliding variable. Next, a controller for a real perturbed plant is designed so that it drives the system's representative point to its reference position on the sliding plane in each step. Therefore, the impact of external disturbances on the system's trajectory is minimized, which leads to a reduction of the necessary control effort. Moreover, thanks to a new reaching law applied in the reference profile generator, the sliding surface shift in each step is strictly limited and a switching type of motion occurs. Finally, under the assumption of boundedness and smoothness of continuous-time disturbance, a compensation scheme is added. It is proved that this control strategy reduces the quasi-sliding mode band-width from $O(T)$ to $O\left(T^{3}\right)$ order from the very beginning of the regulation process. Moreover, it is shown that the maximum state variable errors become of $O\left(T^{3}\right)$ order as well. These achievements directly reduce the energy consumption in the closed-loop system, which is nowadays one of the crucial factors in control engineering.
\end{abstract}

Keywords: control design; discrete-time systems; disturbance compensation; reaching law approach; sliding mode control; trajectory generator

\section{Introduction}

The term sliding mode has first appeared in the theory of variable structure systems [1] and referred to a characteristic form of nonlinear, discontinuous, feedback control, as described in [2,3]. This comprehensive control scheme may be successfully applied to both linear [4] and nonlinear systems [5,6]. In general, the sliding mode control process consists of two stages [7]. In the first stage, the so-called reaching phase, the system's state is driven to a predefined surface in the state space. In the second stage, the so-called sliding phase, the representative point of the system slides along this surface until it reaches the equilibrium point. Various authors discussed the sliding mode design [8,9] and its applications [10] in the literature. Sliding modes are especially widely applied in power electronics, for example, for electronic converters [11,12], electrical motor drives [13], and power systems [14]. The greatest advantage of the sliding mode control is that once the system's state becomes bound to the sliding plane, any matched external disturbances and model uncertainties are immediately rejected [15]. Therefore, in order to improve the system's robustness, it is highly recommended to make the reaching phase as short as possible [16]. However, enforcing faster convergence often requires large magnitudes of 
the control input and therefore, consumes more energy. This observation resulted in the development of time-varying sliding planes.

The time-varying sliding planes have been widely applied for continuous-time systems since the 1990s. Their basic design assumes that the sliding plane must cross the initial position of the system's representative point at time $t=0$ and for any $t>0$ it shifts in the state space, according to different algorithms, until it crosses the system's desired position at $t=t_{0}$. Afterward, the sliding plane remains still. As follows, the sliding variable is defined as the distance between the representative point of the system and the sliding plane, and the controller's task is to keep the system's state on the sliding plane from the very beginning of the control process. Consequently, the reaching phase is completely eliminated and the benefit of insensitivity to external disturbances and modeling uncertainties is observed in the entire course of motion. The first time-varying sliding plane designs combined rotating [17] and shifting surfaces [18], which, for example, were applied in the position control of a DC motor drive [19].

With the rapid development of digital control systems, most of the sliding mode properties have been transferred to the discrete-time domain resulting in the quasi-sliding mode. As shown by Milosavljevic [20], due to delays in the control channel, in the discretetime, the representative point of the system only remains in a certain vicinity of the sliding plane, not on the plane itself, creating the so-called quasi-sliding mode band. The properties [21] and stability of the quasi-sliding mode [22,23] have been studied in numerous further works. Some of the authors concentrated on the equivalent control method [24], others considered the control saturation [25], while another group utilized the Lyapunov function-based design [26]. However, a clear definition of the quasi-sliding mode was first introduced in the seminal study of Gao, Wang, and Homaifa in 1995 [27]. According to their paper, the quasi-sliding mode exists when the sliding plane is reached and crossed in finite time. After the first crossing of the sliding plane, the sign of the sliding variable changes in each step and its absolute value does not exceed an a priori known value. This definition constituted a solid foundation for future quasi-sliding mode research and will be adopted further in this study. The work of Gao et al. [27] also established a family of switching type reaching laws, which provide the characteristic zigzagging motion around the sliding plane. Afterward, many new reaching laws have been introduced, such as $[28,29]$. Their generalized form was presented in [30]. A direct measure of their effectiveness and the system's robustness has become the width of the achieved band. It was proved in [31] that for discretized systems, the ultimate bandwidth directly depends on the discretization period and therefore is of $O(T)$ order.

Along with the development of digital sliding mode control, the issues connected with the energetical efficiency of control systems have come into the spotlight. The optimal control design has grown in popularity [32]. As energy saving is one of the main requirements of nowadays control systems, and especially of power electronics systems, it has become an important problem in the quasi-sliding mode design. The obtained switching type of motion around the sliding plane directly causes larger energy consumption of the closedloop system. Therefore, reducing the width of the quasi-sliding mode band, and as follows reducing the necessary control effort, has become the main area of interest. For that purpose, different authors attempted to design new reaching functions [33], eliminate the chattering effects [34-36], or employ various disturbance compensators [37,38]. However, as in the discrete-time, the controller is unable to keep the system's state exactly on the sliding plane, the idea of time-varying sliding surfaces has not been widely applied in this case.

Sliding mode control has become the center of our efforts in this paper as well. We propose a new time-varying sliding mode controller for sampled-data systems. The originality of this work is manifested in a new, simple but fairly efficient, design of the timevarying sliding surface. It is generated so that the representative point of the system belongs to the surface at the initial step $k=0$. Next, for $0<k<k_{0}$, the sliding plane shifts according to a pre-generated reference sliding variable profile $s_{r}(k)$, monotonically approaching the system's demand position. Once, the demand position is crossed, at $k=k_{0}$, the plane shifts 
by $\pm \beta$ in each step, ensuring the switching requirement, stated by Gao et al. [27]. For the generation of such a sliding surface, a reference sliding variable $s_{r}(k)$ and a new reaching law are utilized. Next, the control signal for the real perturbed system is designed so that it drives the representative point onto the sliding plane at each time instant from the very beginning of the control process. Our study shows that this control scheme eliminates the reaching phase, provides all the quasi-sliding mode properties, as defined in [27], including the switching condition, and minimizes the ultimate band-width. In other words, the proposed control method reduces chattering, which results in a significant reduction of energy consumption in the sliding phase. This achievement is particularly important for the control of electric motor drives, where energetical efficiency is one of the main factors in determining the control scheme. Supplemented by a disturbance compensation scheme, our strategy reduces the width of the achieved quasi-sliding mode band from $O(T)$ to $O\left(T^{3}\right)$ order. Moreover, it is proved that the maximum deviations of all state variables from their demand values become of $O\left(T^{3}\right)$ order as well. In short, our control method improves the robustness of the system in comparison to other control methods, previously proposed in the literature, and increases the energetical efficiency of the closed-loop system.

\section{Problem Formulation}

This paper presents the design of a sliding mode controller for a linear, continuoustime system subject to matched external disturbances

$$
\dot{x}(t)=\boldsymbol{\Phi} x(t)+\boldsymbol{\Gamma} u(t)+\boldsymbol{\Gamma} f(t),
$$

where appropriate symbols represent: $x(t)$-the system's $n \times 1$ state vector, $\boldsymbol{\Phi}$-the $n \times n$ state matrix, $\Gamma$-the $n \times 1$ input vector, $u(t)$-the control signal and $f(t)$-the disturbance. The aim of the control algorithm is to drive the system from its initial state, denoted with $x(0)=x_{0}$, to the desired position $x_{d}$ in finite time. The control is feasible, as we assume that the disturbance $f(t)$ is lower and upper bounded by $\pm f_{\max }$, respectively. Moreover, the study is focused on the case when $f(t)$ is a smooth function of time and satisfies the matching condition, as in relation (1). As it is most common that all state measurements and the control implementation include sample and hold devices in their channels, the system will be analyzed in the discrete-time domain. Therefore, with sampling period $T$, the discretized system becomes

$$
\boldsymbol{x}(k+1)=\boldsymbol{A} \boldsymbol{x}(k)+\boldsymbol{b} u(k)+\boldsymbol{d}(k),
$$

where appropriate matrices are obtained according to the following

$$
\boldsymbol{A}=e^{\boldsymbol{\Phi} T}, \boldsymbol{b}=\int_{0}^{T} e^{\boldsymbol{\Phi} \tau} d \boldsymbol{\tau} \boldsymbol{\Gamma}, \boldsymbol{d}(k)=\int_{0}^{T} e^{\boldsymbol{\Phi} \tau} \boldsymbol{\Gamma} f[(k+1) T-\tau] d \tau .
$$

Equation (3) indicates that the discrete-time system properties directly depend on the choice of the discretization period $T$. Therefore, the obtained input distribution vector $\boldsymbol{b}$, as well as the discretized disturbance $\boldsymbol{d}(k)$, are of $O(T)$ order. Further properties of the discretized disturbance vector have been studied in detail in the noteworthy papers $[31,37]$. With the application of Taylor's series expansion, the disturbance vector $\boldsymbol{d}(k)$ may be expressed as

$$
\boldsymbol{d}(k)=\int_{0}^{T} e^{\boldsymbol{\Phi} \tau} \boldsymbol{\Gamma} f(k T) d \tau+\int_{0}^{T} e^{\boldsymbol{\Phi} \tau} \boldsymbol{\Gamma} v(k T)(T-\tau) d \tau+\int_{0}^{T} e^{\boldsymbol{\Phi} \tau} \boldsymbol{\Gamma} \frac{1}{2 !} w(\mu)(T-\tau)^{2} d \tau+\ldots
$$


where $v(t)=d f(t) / d t$ and $w(t)=d^{2} f(t) / d t^{2}$ are the first and second derivatives of the disturbance and $\mu$ is some moment between the consecutive time steps $k T$ and $(k+1) T$. As presented in [37], from the solution of (4), we get

$$
\boldsymbol{d}(k)=\boldsymbol{b} f(k T)+\frac{1}{2} \boldsymbol{b} v(k T) T+\int_{0}^{T} e^{\boldsymbol{\Phi} \tau} \boldsymbol{\Gamma} \frac{1}{2 !} w(\mu)(T-\tau)^{2} \boldsymbol{d} \tau+\ldots
$$

The first term in (5) proves that the disturbance $d(k)$ linearly depends on the discretization period $T$, as acknowledged before. However, (5) also shows that in the discrete-time domain, the disturbance contains a mismatched term of $O\left(T^{3}\right)$ order, represented by the integral in (5). Finally, $\boldsymbol{d}(k)$ may be approximated with

$$
\boldsymbol{d}(k)=\boldsymbol{b} f(k T)+\frac{1}{2} \boldsymbol{b} v(k T) T+O\left(T^{3}\right) .
$$

Moreover, as the continuous-time disturbance is bounded by $\pm f_{\max }$, the discretized disturbance range is limited as well. Finally, considering the disturbance rate of change we get

$$
\boldsymbol{d}(k)-\boldsymbol{d}(k-1)=O\left(T^{2}\right)
$$

and

$$
\boldsymbol{d}(k)-2 \boldsymbol{d}(k-1)+\boldsymbol{d}(k-2)=O\left(T^{3}\right) .
$$

The aim of this paper is to present a new unconventional quasi-sliding mode design for the presented system. The key idea behind this work is the implementation of a reference sliding variable profile to determine the time-varying sliding surface evolution, which is described in the following chapter. Next, a discrete-time control law for the original system is proposed, which restricts the system's state to the quasi-sliding mode band from the beginning of the control process, therefore eliminating the reaching phase. Finally, we introduce a disturbance compensation scheme, which results in the restriction of the sliding mode bandwidth and the state variable error values to $O\left(T^{3}\right)$ order. As follows, the proposed control scheme reduces the necessary control effort and energy consumption in the whole regulation process. The paper is concluded with a simulation example.

\section{Time-Varying Quasi-SMC}

In this chapter, we propose a new discrete-time sliding mode control strategy based on a time-varying sliding surface. Section 1 presents the generation of the desired sliding surface evolution, employing a reference sliding variable. Next, the control law for the original system (2) is presented and the properties of the resulting sliding mode are analyzed. Finally, in Section 3 an additional compensation scheme for the matched external disturbances is introduced, which further reduces the quasi-sliding mode bandwidth and therefore, reduces the control effort in the sliding stage. The structure of the proposed closed-loop system is presented in Figure 1 below. 


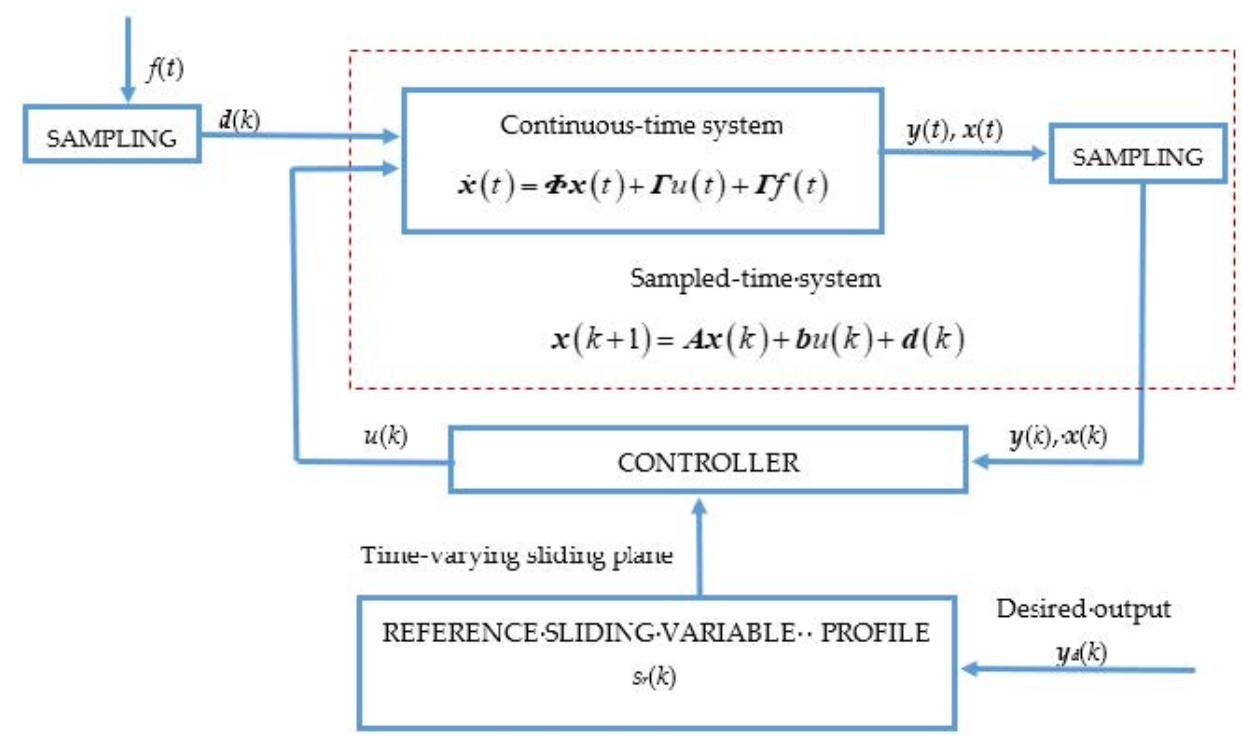

Figure 1. Structure of the closed-loop system.

\subsection{Time-Varying Sliding Plane Design}

The first step in the sliding mode control design is the proper selection of the sliding hyperplane. In order to limit the impact of external disturbances on the system's trajectory, a time-varying sliding surface will be utilized. To eliminate the reaching phase, the surface must be designed so that it crosses the initial position of the system's representative point at $k=0$ and for any $k>0$ it shifts until it crosses the desired state at $k=k_{0}$. Such sliding surface is defined with

$$
c e(k)-s_{r}(k)=0,
$$

where $c$ is an $1 \times n$ control vector, chosen so that $\boldsymbol{c} b \neq 0$ and to ensure stability of the steady state and $\boldsymbol{e}(k)=x_{\boldsymbol{d}}-\boldsymbol{x}(k)$ represents the error vector. The first term in $(9), \boldsymbol{c} \boldsymbol{e}(k)=s(k)$, defines the sliding variable of the system, and $s_{r}(k)$ is its pre-generated reference profile. It combines the reference profiles of all state variables of the system as follows

$$
s_{r}(k)=c\left[x_{d}-x_{r}(k)\right],
$$

where $x_{r}(k)$ represents the reference state vector and $x_{0}=x_{r}(0)$. Therefore, the initial value of the reference sliding variable satisfies $s_{r}(0)=s(0)=c e(0)$, so the system's representative point is on the sliding plane at the beginning of the control process. The sliding variable defined in (9) physically represents the distance between the current position of the representative point of the system and its desired position in the same step. The task of the control scheme is to keep the system's state on the sliding plane through the whole regulation process or, in other words, to steer the system's state vector $x(k)$ to the reference $x_{r}(k)$. From the initial condition, we conclude that at the beginning of the control process for $k=0$, the representative point of the system belongs to the sliding surface. Therefore, the reachability of the desired position $x_{d}$ depends on the sliding surface evolution. As the properties of this surface directly depend on the reference sliding variable profile (10), the algorithm for its proper generation will be discussed next.

As the reference sliding variable profile defines the evolution of the sliding surface, it must begin with the initial condition $s_{r}(0)=c e(0)$ and monotonically approach zero. Moreover, as the switching type definition of the sliding mode should be satisfied, once the reference crosses zero, its sign must change in each consecutive time instant. To provide the 
above properties, we propose to generate the reference sliding variable profile according to a new switching type reaching law

$$
s_{r}(k+1)= \begin{cases}s_{r}(k)-(\alpha+\beta) \operatorname{sgn}\left[s_{r}(k)\right] & \text { for }\left|s_{r}(k)\right|>\alpha \\ -\beta \operatorname{sgn}\left[s_{r}(k)\right] & \text { for }\left|s_{r}(k)\right| \leq \alpha^{\prime}\end{cases}
$$

where $\alpha, \beta>0$ and $\alpha>\beta$. The introduced reaching law ensures unique favorable properties of the sliding variable evolution, such as restriction of its rate of change, finite time convergence to the vicinity of zero, and satisfaction of the switching condition stated by Gao et al. [27]. The motion generated with the reaching law (11) may be divided into two modes. When the sliding variable $s_{r}(k)$ is outside the interval $[-\alpha, \alpha]$, so for $\left|s_{r}(k)\right|>\alpha$, the first formula of the reaching law acts. In that case, from (11) we get

$$
s_{r}(k+1)=\left[\left|s_{r}(k)\right|-(\alpha+\beta)\right] \operatorname{sgn}\left[s_{r}(k)\right] .
$$

Considering that $\alpha+\beta$ is always positive and $\alpha>\beta$ then

$$
\left|s_{r}(k+1)\right|<\left|s_{r}(k)\right| .
$$

For $\left|s_{r}(k)\right|>\alpha+\beta$, the sign of the reference sliding variable remains unchanged, i.e., $\operatorname{sgn}\left[s_{r}(k+1)\right]=\operatorname{sgn}\left[s_{r}(k)\right]$. On the other hand, for $\alpha+\beta>\left|s_{r}(k)\right|>\alpha$, the sign of the reference sliding variable changes for the first time, $\operatorname{so} \operatorname{sgn}\left[s_{r}(k+1)\right]=-\operatorname{sgn}\left[s_{r}(k)\right]$. Moreover, from (12) we conclude that the rate of change of $s_{r}(k)$ is constant and expressed with

$$
\left|s_{r}(k)-s_{r}(k+1)\right|=\alpha+\beta .
$$

Therefore, in the whole reaching phase, when $\left|s_{r}(k)\right|>\alpha$, the reference sliding variable is in each step reduced by a constant value. As follows, according to(9), the sliding surface shifts by a constant distance in each step.

We define $k_{0}$ as the first time instant when the reference sliding variable enters the interval $(-\alpha, \alpha)$. Therefore

$$
\left|s_{r}\left(k_{0}\right)\right|<\alpha
$$

And from (11)

$$
k_{0}=\text { floor }\left(\frac{\left|s_{0}\right|-\alpha}{\alpha+\beta}\right)+1 .
$$

Afterward, for any $k \geq k_{0}$ the second formula of the reaching law acts. In that case, (11) takes the form

$$
\operatorname{sgn}\left[s_{r}(k+1)\right]\left|s_{r}(k+1)\right|=-\beta \operatorname{sgn}\left[s_{r}(k)\right] .
$$

Therefore, not later than between steps $k_{0}$ and $k_{0}+1$, the sign of $s_{r}(k)$ changes for the first time, i.e., $\operatorname{sgn}\left[s_{r}\left(k_{0}+1\right)\right]=-\operatorname{sgn}\left[s_{0}\right]$. Moreover, the shift of the sliding surface between steps $k_{0}$ and $k_{0}+1$ satisfies

$$
\left|s_{r}\left(k_{0}\right)-s_{r}\left(k_{0}+1\right)\right| \leq \alpha+\beta .
$$

As $\beta$ is a positive constant, from (17) we conclude that for any $k \geq k_{0}$ the change of sign occurs, so

$$
\operatorname{sgn}\left[s_{r}(k+1)\right]=-\operatorname{sgn}\left[s_{r}(k)\right]
$$

and

$$
\left|s_{r}(k+1)\right|=\beta,
$$

which fulfills the requirements for the existence of the quasi-sliding mode. Moreover, for $k \geq k_{0}+1$, the rate of change becomes

$$
\left|s_{r}(k)-s_{r}(k+1)\right|=2 \beta .
$$


Taking into account (14), (18), and (21), one notices that the rate of change of $s_{r}(k)$ is limited by $\alpha+\beta$ in the whole process. It may also be concluded that in the reaching phase, the pace of convergence is mainly regulated by the value of $\alpha$ as $\alpha>\beta$. In the sliding phase, the reference sliding variable is, in each step, switched between $\pm \beta$, so $\beta$ should be as small as possible to minimize the chattering problem and the quasi-sliding mode bandwidth.

\subsection{Control Law}

The proposed reference sliding variable profile begins with the initial condition of the original system, $s_{r}(0)=s(0)$, monotonically converges to zero and crosses it in finite time. Furthermore, after the sign of $s_{r}(k)$ changes for the first time, it will change again in each successive time instant and its value will never exceed $\beta$. One may conclude that the reference profile satisfies all the quasi-sliding mode requirements stated by Gao et al. [27]. Consequently, the task of the sliding mode controller for the original system (2) becomes to steer the system's state to the reference position in each step, or in other words to drive the system's representative point onto the sliding plane (9). The required control law may be obtained by assigning

$$
s(k+1)-s_{r}(k+1)=0 .
$$

Substituting (2) into (22) and solving for $u(k)$ we get

$$
u(k)=(\boldsymbol{c} \boldsymbol{b})^{-1}\left[\boldsymbol{c} \boldsymbol{x}_{\boldsymbol{d}}-\boldsymbol{c} \boldsymbol{A} \boldsymbol{x}(k)-s_{r}(k+1)\right],
$$

while the unknown disturbance term is neglected. With the control signal (23), the closedloop system (2) becomes

$$
\boldsymbol{x}(k+1)=\left[\mathbf{1}-\boldsymbol{b}(\boldsymbol{c} \boldsymbol{b})^{-1} \boldsymbol{c}\right] \boldsymbol{A} \boldsymbol{x}(k)+\boldsymbol{b}(\boldsymbol{c} \boldsymbol{b})^{-1} \boldsymbol{c} \boldsymbol{x}_{\boldsymbol{d}}-\boldsymbol{b}(\boldsymbol{c} \boldsymbol{b})^{-1} s_{r}(k+1)+\boldsymbol{d}(k) .
$$

As follows, from (9) and (24), the sliding variable of the closed-loop system equals

$$
s(k+1)=s_{r}(k+1)-c \boldsymbol{d}(k) .
$$

Let the term $c d(k)$ be denoted with $D(k)$. Considering the boundedness of the disturbance term $|f(k)| \leq f_{\max }$, we get

$$
\left|s(k+1)-s_{r}(k+1)\right| \leq D_{\max }=\boldsymbol{c} \boldsymbol{b} f_{\max }
$$

From (26) it may be concluded that the designed sliding mode controller drives the system's representative point to the reference position with an accuracy of the disturbance in each step. Nevertheless, with the appropriate selection of the reference profile, the properties of the quasi-sliding mode may be preserved, which is shown in the following theorem.

Theorem 1. If $\beta>D_{\text {max }}$ then, for any $k \geq k_{0}, \operatorname{sgn}[s(k+1)]=-\operatorname{sgn}[s(k)]$, so the change of sign of $s(k)=c e(k)$ occurs and the absolute value of the sliding variable does not exceed $\beta+D_{\text {max }}$.

Proof of Theorem 1. It has already been shown that $s_{r}(k)$ monotonically converges to zero and crosses it in finite time. Moreover, in the reaching phase (14) holds, so the reference sliding variable decreases in each step by $\alpha+\beta$. Consequently, if $\beta>D_{\max }$, from (26), we conclude that $s(k)$ is monotonically decreasing for any $k<k_{0}$.

The reference $s_{r}(k)$ changes its sign for the first time in finite time, not later than in step $k_{0}+1$. Therefore, $\operatorname{sgn}\left[s_{r}\left(k_{0}+1\right)\right]=-\operatorname{sgn}\left(s_{0}\right)$ and for any $k \geq k_{0}$, (19) and (20) hold. Consequently, from (25), for any $k \geq k_{0}$ we get:

$$
\operatorname{sgn}[s(k+1)]|s(k+1)|=\beta \operatorname{sgn}\left[s_{r}(k+1)\right]-D(k) .
$$


The sign of $D(k)$ is unknown, however, taking into account (26), we conclude that when $\beta>D_{\max }$, then $\operatorname{sgn}[s(k+1)]=\operatorname{sgn}\left[s_{r}(k+1)\right]=-\operatorname{sgn}[s(k)]$. Moreover, considering (20), we notice that for any $k \geq k_{0}+1$,

$$
|s(k)| \leq \beta+D_{\max } .
$$

which concludes the proof.

We have shown that, for any $k \geq k_{0}+1$, the switching type quasi-sliding mode exists. The sliding variable is driven to the interval of the width $2\left(\beta+D_{\max }\right)$. As $\beta$ determines the quasi-sliding mode bandwidth and is only limited by $\beta>D_{\max }$, it shall be chosen as the smallest admissible value, which results in the bandwidth of $O(T)$ order. We must also acknowledge that the smaller $\beta$ becomes, the smaller the necessary control effort. As the shift of the sliding surface is strictly limited in each step, the proposed control method guarantees a restriction of the rate of change of the sliding variable. Taking into account the rate of change of the reference sliding variable, for any $k \leq k_{0}$, we get

$$
|s(k)-s(k+1)| \leq \alpha+\beta+2 D_{\max }
$$

and for any $k \geq k_{0}+1$

$$
|s(k)-s(k+1)| \leq 2 \beta+2 D_{\max } .
$$

The restriction of the rate of change of the sliding variable results in a restriction of the necessary control effort and therefore, it increases the energetical efficiency of the closed-loop system.

\section{3. $O\left(T^{3}\right)$ Accuracy}

In this section, in order to improve the robustness of the system, we supplement our control strategy with a disturbance compensation algorithm. The compensation term is constructed from the current and past state measurements and past control signal value in order to calculate the past disturbance impact $D(k-1)$ and $D(k-2)$, as follows

$$
\begin{gathered}
D(k-1)=\boldsymbol{c}[\boldsymbol{x}(k)-\boldsymbol{A} \boldsymbol{x}(k-1)-\boldsymbol{b} u(k-1)], \\
D(k-2)=\boldsymbol{c}[\boldsymbol{x}(k-1)-\boldsymbol{A} \boldsymbol{x}(k-2)-\boldsymbol{b} u(k-2)] .
\end{gathered}
$$

As the disturbance $f(t)$ is a continuous function of time, the rate of change of the discretized disturbance vector $\boldsymbol{d}(k)$ is limited, as noticed in (7) and (8). Therefore, its impact on the sliding variable is also limited, which, using (8), may be described with

$$
|D(k)-2 D(k-1)+D(k-2)| \leq \Delta D .
$$

The obtained previous disturbance values may be used as an estimate for the current disturbance $D(k)$ in the revised reaching law

$$
s(k+1)-s_{r}(k+1)=-D(k)+2 D(k-1)-D(k-2) .
$$

According to (8), the error of such estimation remains of $O\left(T^{3}\right)$ order. With the application of (33) to system (2), we get the control signal

$$
u(k)=(\boldsymbol{c} \boldsymbol{b})^{-1}\left[\boldsymbol{c} x_{\boldsymbol{d}}-\boldsymbol{c A x}(k)-s_{r}(k+1)-2 D(k-1)+D(k+2)\right] .
$$

The estimation of the current disturbance value is a dynamic process. Therefore, the implementation of the disturbance compensator introduces an additional state variable to the analyzed system. With the additional dynamics, the problem may be described with the following set of equations 


$$
\begin{gathered}
x(k+1)=A x(k)+\boldsymbol{b} u(k)+2 \boldsymbol{d}(k-1)-\boldsymbol{d}(k-2) \\
u(k+1)=(\boldsymbol{c b})^{-1}\left[\boldsymbol{c} \boldsymbol{x}_{\boldsymbol{d}}-\boldsymbol{c} \boldsymbol{A} \boldsymbol{x}(k+1)-2 \boldsymbol{c d}(k-1)+\boldsymbol{c d}(k-2)\right] .
\end{gathered}
$$

From the implementation of (35) in (36) one gets

$$
u(k+1)=(\boldsymbol{c} \boldsymbol{b})^{-1} \boldsymbol{c} x_{\boldsymbol{d}}+\left[\begin{array}{ll}
-(\boldsymbol{c} \boldsymbol{b})^{-1} \boldsymbol{c} \boldsymbol{A}^{2} & -(\boldsymbol{c} \boldsymbol{b})^{-1} \boldsymbol{c} \boldsymbol{A} \boldsymbol{b}
\end{array}\right]\left[\begin{array}{l}
\boldsymbol{x}(k) \\
u(k)
\end{array}\right]-(\boldsymbol{c} \boldsymbol{b})^{-1} \boldsymbol{c}\left(\boldsymbol{A}+\mathbf{1}_{n \times n}\right)[2 \boldsymbol{d}(k-1)-\boldsymbol{d}(k-2)] .
$$

Therefore, the closed-loop system is described with the following state equation

$$
\left[\begin{array}{l}
x(k+1) \\
u(k+1)
\end{array}\right]=\widehat{A}\left[\begin{array}{l}
x(k) \\
u(k)
\end{array}\right]+\left[\begin{array}{c}
\mathbf{1}_{n \times n} \\
-(\boldsymbol{c} \boldsymbol{b})^{-1} \boldsymbol{c}\left(\boldsymbol{A}+\mathbf{1}_{n \times n}\right)
\end{array}\right][2 \boldsymbol{d}(k-1)-\boldsymbol{d}(k-2)]+\left[\begin{array}{c}
\mathbf{0}_{n \times 1} \\
(\boldsymbol{c} \boldsymbol{b})^{-1} \boldsymbol{c} \boldsymbol{x}_{\boldsymbol{d}}
\end{array}\right],
$$

where the state matrix is

$$
\widehat{A}=\left[\begin{array}{cc}
A & b \\
-(\boldsymbol{c} b)^{-1} \boldsymbol{c} A^{2} & -(\boldsymbol{c} \boldsymbol{b})^{-1} \boldsymbol{c} \boldsymbol{A} \boldsymbol{b}
\end{array}\right] .
$$

Let us denote the eigenvalues of the system (38) with $z_{i}$, where $i=1, \ldots, n+1$. With this notation the characteristic polynomial of the closed-loop system becomes

$$
0=\operatorname{det}\left(\mathbf{1}_{(n+1) \times(n+1)} z_{i}-\widehat{A}\right)=\left|\begin{array}{cc}
\mathbf{1}_{n \times n} z_{i}-\boldsymbol{A} & -\boldsymbol{b} \\
(\boldsymbol{c} \boldsymbol{b})^{-1} \boldsymbol{c} \boldsymbol{A}^{2} & z_{i}+(\boldsymbol{c} \boldsymbol{b})^{-1} \boldsymbol{c} \boldsymbol{A} \boldsymbol{b}
\end{array}\right| .
$$

To determine the eigenvalues $z_{i}$ of the state matrix (39), the following similarity transformation may be utilized

$$
\widehat{A}=\left[\begin{array}{cc}
A & \boldsymbol{b} \\
-(\boldsymbol{c} \boldsymbol{b})^{-1} \boldsymbol{c} A^{2} & -(\boldsymbol{c} \boldsymbol{b})^{-1} \boldsymbol{c} A \boldsymbol{b}
\end{array}\right]=\boldsymbol{M}^{-1}\left[\begin{array}{cc}
A_{c} & \boldsymbol{b} \\
0_{1 \times n} & 0
\end{array}\right] M,
$$

where $A_{c}=\left[\mathbf{1}_{n \times n}-\boldsymbol{b}(\boldsymbol{c b})^{-1} \boldsymbol{c}\right] \boldsymbol{A}$ and $\boldsymbol{M}$ is an invertible matrix such that

$$
\boldsymbol{M}=\left[\begin{array}{cc}
\mathbf{1}_{n \times n} & \mathbf{0}_{n \times 1} \\
(\boldsymbol{c} \boldsymbol{b})^{-1} \boldsymbol{c A} & 1
\end{array}\right]
$$

and

$$
\boldsymbol{M}^{-1}=\left[\begin{array}{cc}
\mathbf{1}_{n \times n} & 0_{n \times 1} \\
-(\boldsymbol{c} \boldsymbol{b})^{-1} \boldsymbol{c A} & 1
\end{array}\right] .
$$

From the multiplication of (41) we get

$$
\begin{aligned}
& \boldsymbol{M}^{-1}\left[\begin{array}{cc}
\boldsymbol{A}_{\boldsymbol{c}} & \boldsymbol{b} \\
\mathbf{0}_{1 \times n} & 0
\end{array}\right] \boldsymbol{M}=\left[\begin{array}{cc}
\mathbf{1}_{n \times n} & \mathbf{0}_{n \times 1} \\
-(\boldsymbol{c} \boldsymbol{b})^{-1} \boldsymbol{c A} & 1
\end{array}\right]\left[\begin{array}{cc}
\left(\mathbf{1}_{n \times n}-\boldsymbol{b}(\boldsymbol{c b})^{-1} \boldsymbol{c}\right) \boldsymbol{A} & \boldsymbol{b} \\
\mathbf{0}_{1 \times n} & 0
\end{array}\right]\left[\begin{array}{cc}
\mathbf{1}_{n \times n} & \mathbf{0}_{n \times 1} \\
(\boldsymbol{c b})^{-1} \boldsymbol{c A} & 1
\end{array}\right]= \\
& {\left[\begin{array}{cc}
\left(\mathbf{1}_{n \times n}-\boldsymbol{b}(\boldsymbol{c b})^{-1} \boldsymbol{c}\right) \boldsymbol{A} & \boldsymbol{b} \\
-(\boldsymbol{c} \boldsymbol{b})^{-1} \boldsymbol{c A}\left(\mathbf{1}_{n \times n}-\boldsymbol{b}(\boldsymbol{c b})^{-1} \boldsymbol{c}\right) A & -(\boldsymbol{c} \boldsymbol{b})^{-1} \boldsymbol{c A b}
\end{array}\right]\left[\begin{array}{cc}
\mathbf{1}_{n \times n} & 0_{n \times 1} \\
(\boldsymbol{c b})^{-1} c \boldsymbol{c} & 1
\end{array}\right]=\left[\begin{array}{cc}
A & \boldsymbol{b} \\
-(\boldsymbol{c} \boldsymbol{b})^{-1} \boldsymbol{c} A^{2} & -(\boldsymbol{c} \boldsymbol{b})^{-1} \boldsymbol{c} A \boldsymbol{b}
\end{array}\right]=\widehat{A} \text {. }}
\end{aligned}
$$

From (44) it follows that

$$
z_{1, \ldots, n}=\operatorname{eig}\left\{\left[\mathbf{1}_{n \times n}-\boldsymbol{b}(\boldsymbol{c} \boldsymbol{b})^{-1} \boldsymbol{c}\right] \boldsymbol{A}\right\} \wedge z_{n+1}=0 .
$$

Equation (45) indicates that the placement of eigenvalues $z_{1}, \ldots, z_{n}$ depends on the choice of the sliding plane. The dynamics of the disturbance compensator introduced one extra eigenvalue $z_{n+1}$ located at the origin of the complex plane. Therefore, the compensator's dynamics are stable and the whole system's stability depends on the choice of vector $c$ only. However, this modified control scheme enforces a modification of the 
chosen control parameters in order to provide minimal width of the quasi-sliding mode band and consequently reduce chattering effects.

Theorem 2. If $\beta>\Delta D$ then, for any $k \geq k_{0}$

$$
\operatorname{sgn}[s(k+1)]=-\operatorname{sgn}[s(k)]
$$

and the sliding variable satisfies

$$
|s(k)| \leq \beta+\Delta D \text {. }
$$

Proof of Theorem 2. In Section 1 of this chapter, we have shown that

- the reference sliding variable $s_{r}(k)$ changes its sign for the first time not later than in $k_{0}+1$,

- for any $k \geq k_{0} \operatorname{sgn}\left[s_{r}(k+1)\right]=-\operatorname{sgn}\left[s_{r}(k)\right]$,

- for any $k \geq k_{0}+1$, the reference satisfies: $\left|s_{r}(k)\right|=\beta$.

Therefore, the reference sliding variable satisfies all the properties of the quasi-sliding mode as specified in [27]. In the reaching phase, for any $k<k_{0}$, the reference sliding variable approaches zero by $\alpha+\beta$ in each step. Considering (32), when $\alpha+\beta>\Delta D$, then (33) leads to the conclusion that $s(k)$ is decreasing by minimum $\alpha+\beta-\Delta D$ for any $k<k_{0}$. Considering the properties of $s_{r}(k)$, for any $k \geq k_{0}$ from (33) we get

$$
\operatorname{sgn}[s(k+1)]|s(k+1)|=\beta \operatorname{sgn}\left[s_{r}(k+1)\right]-D(k)+2 D(k-1)-D(k-2) .
$$

Taking into account (32), we conclude that if $\beta>\Delta D$, then $\operatorname{sgn}[s(k+1)]=\operatorname{sgn}\left[s_{r}(k+1)\right]$ and

$$
|s(k+1)| \leq \beta+\Delta D .
$$

As (19) holds for any $k \geq k_{0}$, then (46) is satisfied as well, which completes the proof.

We have shown that the enhanced version of the controller ensures the existence of the quasi-sliding mode with the ultimate bandwidth of $2(\beta+\Delta D)$. We notice that the parameter $\beta$ is directly responsible for the width of the quasi-sliding mode band and it is only constrained by $\beta>\Delta D$. Therefore, knowing the bounds of the disturbance, one may always select $\beta$ as the smallest admissible value. As follows, $\beta$ becomes of $O\left(T^{3}\right)$ order. Considering that the second-order disturbance difference is of $O\left(T^{3}\right)$ order, as defined in (8), the width of the whole quasi-sliding mode band becomes of $O\left(T^{3}\right)$ order and its minimum values is $4 \Delta D$. This clearly shows that with the reduction of the discretization period, the control error resulting from chattering effects is significantly reduced, which leads to limitation of the control effort and energy consumption in the sliding phase. In this control algorithm, the rate of change of the sliding variable, for any $k \leq k_{0}$, is restricted by

$$
|s(k)-s(k+1)| \leq \alpha+\beta+2 \Delta D
$$

and, for any $k \geq k_{0}+1$ by

$$
|s(k)-s(k+1)| \leq 2 \beta+2 \Delta D .
$$

Restraining the rate of change of the sliding variable directly results in the reduction of the control magnitudes in the reaching phase, which leads to better energetical efficiency of the closed-loop system.

Once we have shown that the application of the proposed disturbance compensator ensures a reduction of the quasi-sliding mode bandwidth to order $O\left(T^{3}\right)$, we may now proceed to estimate the maximum state variable error values. First, we will concentrate on the stage when the sliding surface approaches the desired position. It will be shown 
that the state variables deviations from their reference values in that stage are maximum of $O\left(T^{3}\right)$ order. Next, we will derive the maximum error values for all the system's state variables, while the sliding surface oscillates around the steady-state. As the choice of the sliding plane is arbitrary, in the following calculations it is assumed that

$$
z_{1, \ldots, n}=\operatorname{eig}\left\{\left[\mathbf{1}_{n \times n}-\boldsymbol{b}(\boldsymbol{c} \boldsymbol{b})^{-1} \boldsymbol{c}\right] \boldsymbol{A}\right\}=0,
$$

which implies that all the poles are located at the origin of the complex plane.

Theorem 3. For systems controlled according to (34), in the reaching phase

$$
\left|\Delta x_{i}(k)\right|=\left|p_{\mathbf{i}}\left[x(k)-x_{\mathbf{r}}(k)\right]\right|=O\left(T^{3}\right),
$$

where $\Delta x_{i}$ is the $i$-th state variable deviation from the reference value, $x_{r}(k)$ represents the reference state vector and $p_{i}$ is a row vector such that

$$
p_{\mathrm{i}}=\left[\begin{array}{ccccccc}
0 & \ldots & 0 & 1 & 0 & \ldots & 0 \\
& j & & & & j & \\
& i-1 & & & & n-i &
\end{array}\right] .
$$

Moreover, if vector $c$ is selected so that (52) holds, then the state error in the quasi-sliding mode for any $j \geq k_{0}+n$, is limited as follows

$$
\left|e_{i}(j)\right| \leq \boldsymbol{p}_{\mathbf{i}}\left|x_{\boldsymbol{d}}-\sum_{l=0}^{n-1} \boldsymbol{A}_{\boldsymbol{c}}{ }^{l} \boldsymbol{b}(\boldsymbol{c} \boldsymbol{b})^{-1} \boldsymbol{c} x_{\boldsymbol{d}}\right|+(\beta+\Delta D) \sum_{l=0}^{n-1}\left|\boldsymbol{p}_{\mathbf{i}} \boldsymbol{A}_{\boldsymbol{c}}{ }^{l} \boldsymbol{b}(\boldsymbol{c} \boldsymbol{b})^{-1}\right|,
$$

where $e_{i}$ denotes the $i$-th state variable error $e_{i}(j)=x_{d i}-x_{i}(j)$.

Proof of Theorem 3. To begin the proof, let us introduce the following notation for $d(k)$

$$
\boldsymbol{d}(k)=\boldsymbol{b} g(k)+O\left(T^{3}\right),
$$

where $g(k)$ is the sum of matched disturbance effects from (6) and the mismatched disturbance term is of order $O\left(T^{3}\right)$. Next, using (32) and (33), we describe the absolute sliding variable deviation from the reference with

$$
\left|s(k+1)-s_{r}(k+1)\right|=|D(k)-2 D(k-1)+D(k-2)| \leq \Delta D .
$$

Following (9) and (10), we transform (57) to

$$
\left|\boldsymbol{c}\left[\boldsymbol{x}_{\mathbf{r}}(k+1)-\boldsymbol{x}(k+1)\right]\right|=|\boldsymbol{c}[\boldsymbol{d}(k)-2 \boldsymbol{d}(k-1)+\boldsymbol{d}(k-2)]| \leq \Delta D=O\left(T^{3}\right) .
$$

Considering that vector $c$ is of $O(1)$ order, one may conclude that the $i$-th state variable deviation from the reference is

$$
\left|p_{\mathrm{i}}\left[x(k)-x_{\mathbf{r}}(k)\right]\right|=O\left(T^{3}\right),
$$

which confirms (53). Next, we proceed to calculate $e(k+1)$ from (2) with the control input (34), which yields

$$
\boldsymbol{e}(k+1)=x_{\boldsymbol{d}}-\boldsymbol{A x}(k)-\boldsymbol{b}(\boldsymbol{c} \boldsymbol{b})^{-1} \boldsymbol{c} x_{\boldsymbol{d}}+\boldsymbol{b}(\boldsymbol{c} \boldsymbol{b})^{-1} \boldsymbol{c} \boldsymbol{A} \boldsymbol{x}(k)+\boldsymbol{b}(\boldsymbol{c} \boldsymbol{b})^{-1} s_{r}(k+1)-\boldsymbol{b}(\boldsymbol{c} \boldsymbol{b})^{-1} D(k-2)+2 \boldsymbol{b}(\boldsymbol{c} \boldsymbol{b})^{-1} D(k-1)-\boldsymbol{d}(k) .
$$

Considering that $A_{c}=\left[\mathbf{1}_{n \times n}-\boldsymbol{b}(\boldsymbol{c b})^{-1} c\right] A$, we transform (60) to

$$
\boldsymbol{e}(k+1)=x_{\boldsymbol{d}}-\boldsymbol{A}_{\boldsymbol{c}}{ }^{0} \boldsymbol{b}(\boldsymbol{c} \boldsymbol{b})^{-1} \boldsymbol{x}_{\boldsymbol{d}}-\boldsymbol{A}_{\boldsymbol{c}}{ }^{1} \boldsymbol{x}(k)+\boldsymbol{A}_{\boldsymbol{c}}^{0} \boldsymbol{b}(\boldsymbol{c} \boldsymbol{b})^{-1} s_{r}(k+1)+\boldsymbol{A}_{\boldsymbol{c}}{ }^{0} \boldsymbol{b}(\boldsymbol{c} \boldsymbol{b})^{-1}[2 D(k-1)-D(k-2)]-\boldsymbol{A}_{\mathcal{c}}^{0} \boldsymbol{d}(k) .
$$


Following the same pattern, we write the state error in step $k+2$

$$
\begin{gathered}
\boldsymbol{e}(k+2)=\boldsymbol{x}_{\boldsymbol{d}}-\boldsymbol{A}_{\boldsymbol{c}}^{0} \boldsymbol{b}(\boldsymbol{c} \boldsymbol{b})^{-1} \boldsymbol{c} \boldsymbol{x}_{\boldsymbol{d}}-\boldsymbol{A}_{\boldsymbol{c}}{ }^{1} \boldsymbol{b}(\boldsymbol{c} \boldsymbol{b})^{-1} \boldsymbol{c} x_{\boldsymbol{d}}-\boldsymbol{A}_{\boldsymbol{c}}{ }^{2} \boldsymbol{x}(k)+\boldsymbol{A}_{\boldsymbol{c}}^{1} \boldsymbol{b}(\boldsymbol{c} \boldsymbol{b})^{-1} s_{r}(k+1)+ \\
+\boldsymbol{A}_{\boldsymbol{c}}{ }^{1} \boldsymbol{b}(\boldsymbol{c} \boldsymbol{b})^{-1}[2 D(k-1)-D(k-2)]-\boldsymbol{A}_{\boldsymbol{c}}{ }^{1} \boldsymbol{d}(k)+\boldsymbol{A}_{\boldsymbol{c}}^{0} \boldsymbol{b}(\boldsymbol{c} \boldsymbol{b})^{-1} s_{r}(k+2)+\boldsymbol{A}_{\boldsymbol{c}}^{0} \boldsymbol{b}(\boldsymbol{c} \boldsymbol{b})^{-1}[2 D(k)-D(k-1)]-\boldsymbol{A}_{c}^{0} \boldsymbol{d}(k+1) .
\end{gathered}
$$

In general, for any $j \geq 0$, the state error vector $e(j)$ is

$$
\begin{gathered}
\boldsymbol{e}(j)=x_{\boldsymbol{d}}-\sum_{l=0}^{j-k-1} \boldsymbol{A}_{\boldsymbol{c}}{ }^{l} \boldsymbol{b}(\boldsymbol{c} \boldsymbol{b})^{-1} \boldsymbol{c} \boldsymbol{x}_{\boldsymbol{d}}-\boldsymbol{A}_{\boldsymbol{c}}{ }^{j-k} \boldsymbol{x}(k)+ \\
+\sum_{l=0}^{j-k-1} \boldsymbol{A}_{\boldsymbol{c}}{ }^{l}\left\{\boldsymbol{b}(\boldsymbol{c} \boldsymbol{b})^{-1} s_{r}(j-l)+\boldsymbol{b}(\boldsymbol{c} \boldsymbol{b})^{-1}[2 D(j-l-2)-D(j-l-3)]-\boldsymbol{d}(j-l-1)\right\} .
\end{gathered}
$$

One may notice that when (52) holds, then the state matrix $\boldsymbol{A}_{\boldsymbol{c}}$ is nilpotent, so $\boldsymbol{A}_{\boldsymbol{c}}{ }^{j-k}=0$ for any $j-k \geq n$. Therefore, for any $j \geq k+n$, the sums in (63) may be shortened to $n$ elements

$$
\boldsymbol{e}(j)=x_{\boldsymbol{d}}-\sum_{l=0}^{n-1} \boldsymbol{A}_{\boldsymbol{c}}{ }^{l} \boldsymbol{b}(\boldsymbol{c} \boldsymbol{b})^{-1} \boldsymbol{c} \boldsymbol{x}_{\boldsymbol{d}}+\sum_{l=0}^{n-1} \boldsymbol{A}_{\boldsymbol{c}}{ }^{l}\left\{\boldsymbol{b}(\boldsymbol{c} \boldsymbol{b})^{-1} s_{r}(j-l)+\boldsymbol{b}(\boldsymbol{c} \boldsymbol{b})^{-1}[2 D(j-l-2)-D(j-l-3)]-\boldsymbol{d}(j-l-1)\right\} .
$$

Moreover, from (56), we may derive that

$$
\boldsymbol{b}(\boldsymbol{c} \boldsymbol{b})^{-1} D(k)=\boldsymbol{b}(\boldsymbol{c} \boldsymbol{b})^{-1} \boldsymbol{c}\left[\boldsymbol{b} g(k)+O\left(T^{3}\right)\right]=\boldsymbol{b} g(k)+O\left(T^{3}\right)
$$

Taking into account (56), (65), and the reaching law (33), we notice that

$$
\boldsymbol{b}(\boldsymbol{c} \boldsymbol{b})^{-1} s_{r}(j-l)+\boldsymbol{b}(\boldsymbol{c} \boldsymbol{b})^{-1}[2 D(j-l-2)-D(j-l-3)]-\boldsymbol{d}(j-l-1)=\boldsymbol{b}(\boldsymbol{c} \boldsymbol{b})^{-1} s(j-l)+O\left(T^{3}\right) \text {. }
$$

With the application of (66), we transform (64) to the following form

$$
\boldsymbol{e}(j)=\boldsymbol{x}_{\boldsymbol{d}}-\sum_{l=0}^{n-1} \boldsymbol{A}_{\boldsymbol{c}}{ }^{l} \boldsymbol{b}(\boldsymbol{c} \boldsymbol{b})^{-1} \boldsymbol{c} \boldsymbol{x}_{\boldsymbol{d}}+\sum_{l=0}^{n-1} \boldsymbol{A}_{\boldsymbol{c}}{ }^{l} \boldsymbol{b}(\boldsymbol{c} \boldsymbol{b})^{-1} s(j-l)+O\left(T^{3}\right) \text {. }
$$

Next, we multiply both sides of (67) with the row vector $p_{i}$ obtaining the $i$-th variable's error

$$
\boldsymbol{e}_{i}(j)=\boldsymbol{x}_{\boldsymbol{d} i}-\sum_{l=0}^{n-1} \boldsymbol{p}_{\mathbf{i}} \boldsymbol{A}_{\boldsymbol{c}}{ }^{l} \boldsymbol{b}(\boldsymbol{c} \boldsymbol{b})^{-1} \boldsymbol{c} \boldsymbol{x}_{\boldsymbol{d}}-\sum_{l=0}^{n-1} \boldsymbol{p}_{\mathbf{i}} \boldsymbol{A}_{\boldsymbol{c}}{ }^{l} \boldsymbol{b}(\boldsymbol{c} \boldsymbol{b})^{-1} s(j-l)+O\left(T^{3}\right) .
$$

Equation (68) shows that in each step $j$, where $j \geq k+n$ and $k \geq 0$, all state errors may be calculated using the $n$ previous sliding variable values, $s(j-n+1), s(j-n+2), \ldots, s(j)$. Considering that for any $k \geq k_{0}+1$ the value of the sliding variable is bounded by (49), we conclude that the state errors are bounded as well. From (49) and (68), for any $j \geq k_{0}+n$, we obtain

$$
\left|e_{i}(j)\right| \leq\left|\boldsymbol{x}_{\boldsymbol{d} i}-\sum_{l=0}^{n-1} \boldsymbol{p}_{\mathbf{i}} \boldsymbol{A}_{\boldsymbol{c}}{ }^{l} \boldsymbol{b}(\boldsymbol{c} \boldsymbol{b})^{-1} \boldsymbol{c} \boldsymbol{x}_{\boldsymbol{d}}\right|+(\beta+\Delta D) \sum_{l=0}^{n-1}\left|\boldsymbol{p}_{\mathbf{i}} \boldsymbol{A}_{\boldsymbol{c}}{ }^{l} \boldsymbol{b}(\boldsymbol{c} \boldsymbol{b})^{-1}\right|+O\left(T^{3}\right)
$$

which ends the proof.

Bearing in mind (8), we remember that $\Delta D$ is of $O\left(T^{3}\right)$ order. Moreover, it has already been shown that the width of the quasi-sliding mode band directly depends on the choice of parameter $\beta$. Therefore, $\beta$ must be selected as a function of $T^{3}$ to provide the best robustness and lowest energy costs. Consequently, from (69), one may notice that the maximum state error in the sliding phase is of order $O\left(T^{3}\right)$ as well. 


\section{Simulation Example}

To conclude this study, a simulation example will be analyzed. We design the sliding mode controller for a simple third-order plant, described with the following state equation

$$
\dot{x}(t)=\left[\begin{array}{lll}
0 & 1 & 0 \\
0 & 0 & 1 \\
0 & 0 & 0
\end{array}\right] x(t)+\left[\begin{array}{l}
0 \\
0 \\
1
\end{array}\right][u(t)+f(t)],
$$

The initial conditions are $x(0)=\left[\begin{array}{lll}10 & 0 & 0\end{array}\right]^{\mathrm{T}}$ and the desired state is $x_{d}=\left[\begin{array}{lll}0 & 0 & 0\end{array}\right]^{\mathrm{T}}$. In this example, the disturbance $f(t)$ is a sinusoidal function of time with a frequency of $50 \mathrm{~Hz}$ and an amplitude of 1 . We aim to control the system digitally. Therefore, we discretize the plant with the discretization period $T=1 \mathrm{~ms}$, obtaining

$$
\boldsymbol{x}(k+1)=\boldsymbol{A x}(k)+\boldsymbol{b} u(k)+\boldsymbol{d}(k),
$$

where

$$
A=\left[\begin{array}{ccc}
1 & 0.001 & 5 \cdot 10^{-7} \\
0 & 1 & 0.001 \\
0 & 0 & 1
\end{array}\right], \boldsymbol{b}=\left[\begin{array}{c}
\frac{1}{6} \cdot 10^{-9} \\
5 \cdot 10^{-7} \\
0.001
\end{array}\right] .
$$

The eigenvalues of matrix $A$ in (72) are $z_{1}=z_{2}=z_{3}=1$, so the system is on the verge of stability. In the first step of the control design, we select the sliding plane according to (52). Therefore, we set $c=\left[10.0011 / 3 \times 10^{-6}\right]$, which places all the poles at the origin of the complex plane and therefore, ensures the stability of the closed-loop system. The disturbance $f(t)$ acts through the control channel and changes between \pm 1 , so the maximum disturbance impact is $D_{\max }=1 \times 10^{-9}$ and $\Delta D=0.98 \times 10^{-10}$. Therefore, according to theorem 2, we choose the control parameter $\beta=10^{-10}$. On the other hand, the parameter $\alpha$ is chosen in order to ensure a control limitation of $3 \times 10^{6}$, so $\alpha=3 \times 10^{-3}$. To present the benefits of our control method we compare it with the seminal strategy of Gao et al. [27], where the control signal was obtained with the reaching law

$$
s(k+1)=(1-q T) s(k)-\varepsilon \operatorname{sgn}[s(k)]-D(k) .
$$

For the original strategy we set $q T=3 \times 10^{-4}$ and $\varepsilon=6.67 \times 10^{-6}$, to ensure fulfillment of the same control constraint. The results of our simulations are presented in Figures 1-13.

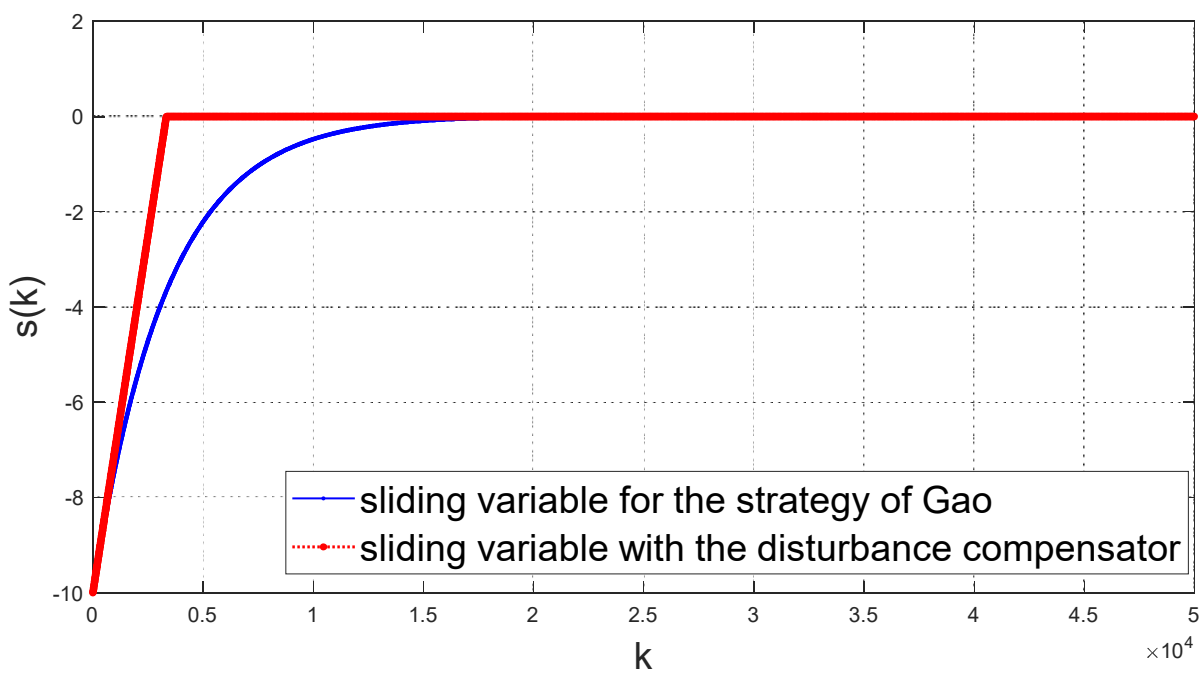

Figure 2. Comparison of the discrete-time sliding variables. 


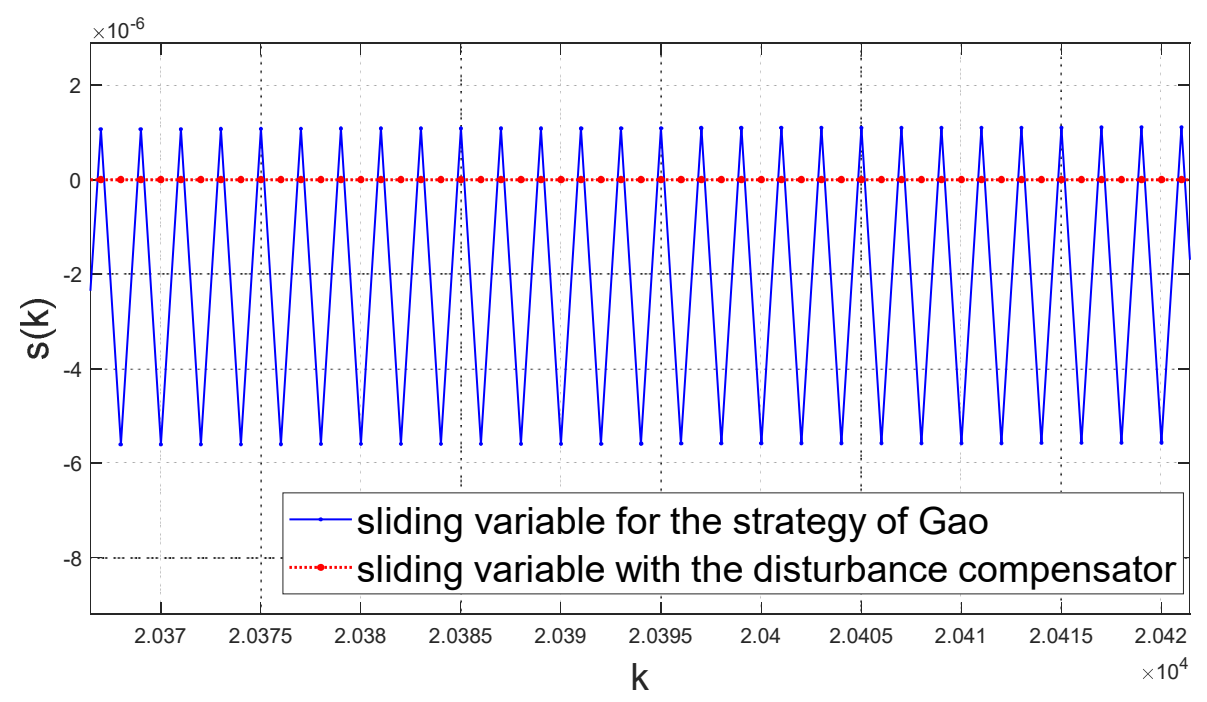

Figure 3. Comparison of the discrete-time sliding variables in the sliding phase.

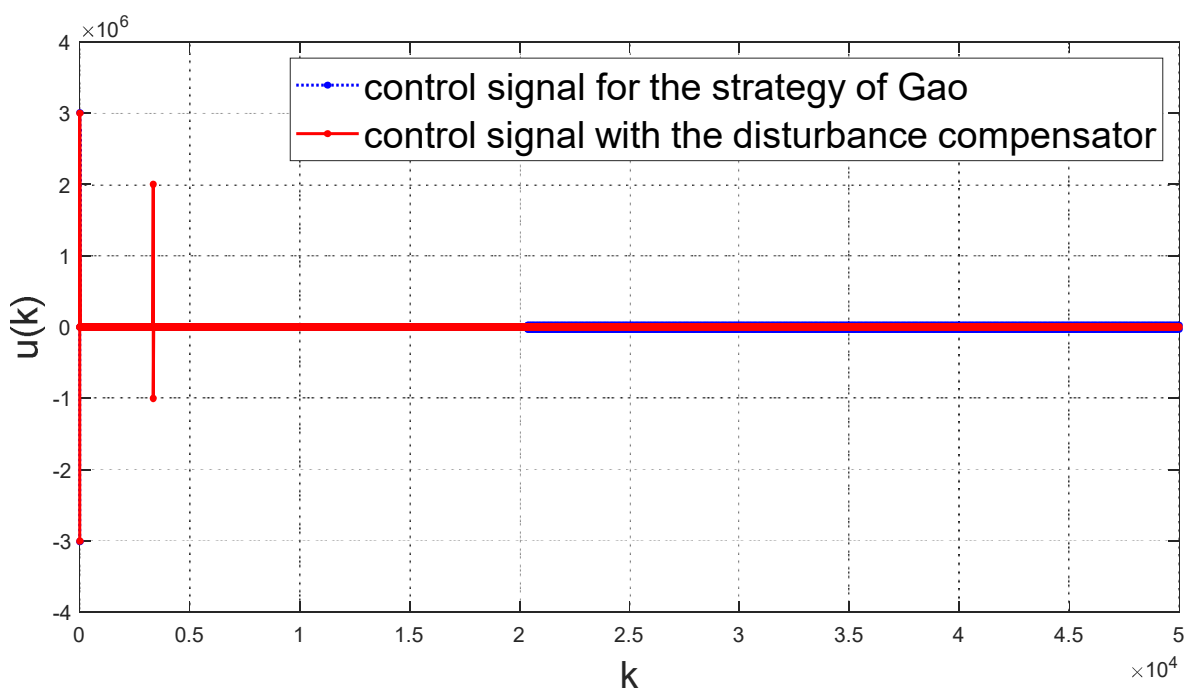

Figure 4. Evolution of the control signal $u(k)$.

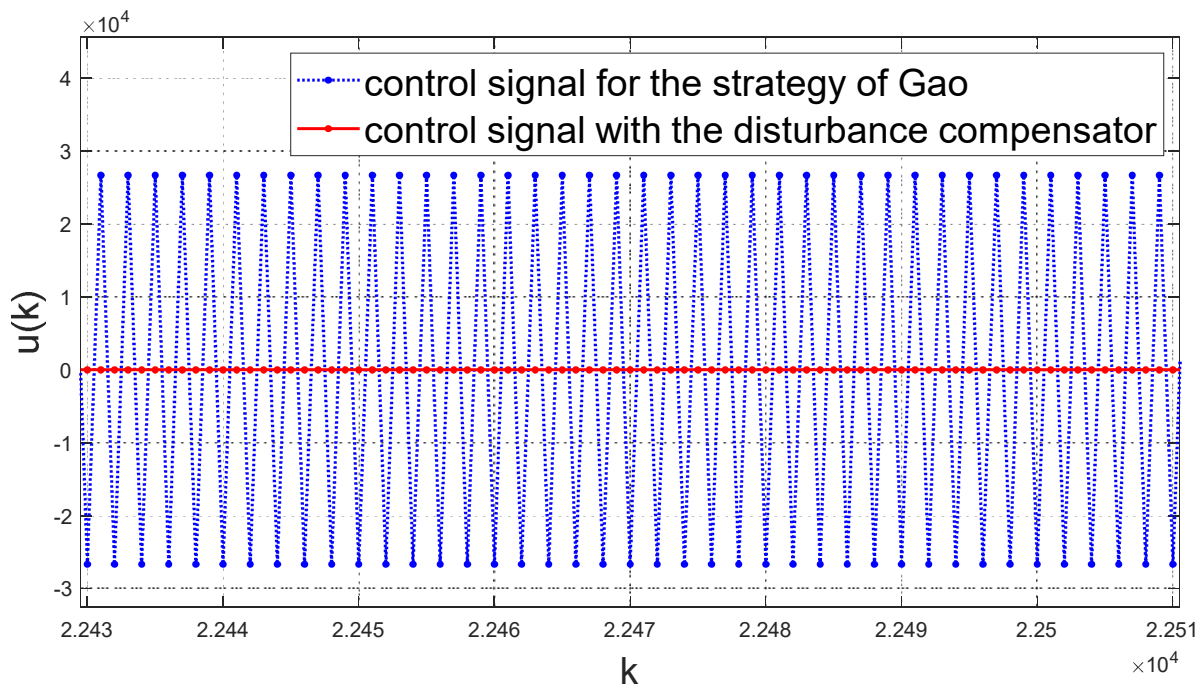

Figure 5. Evolution of the control signal in the sliding phase. 


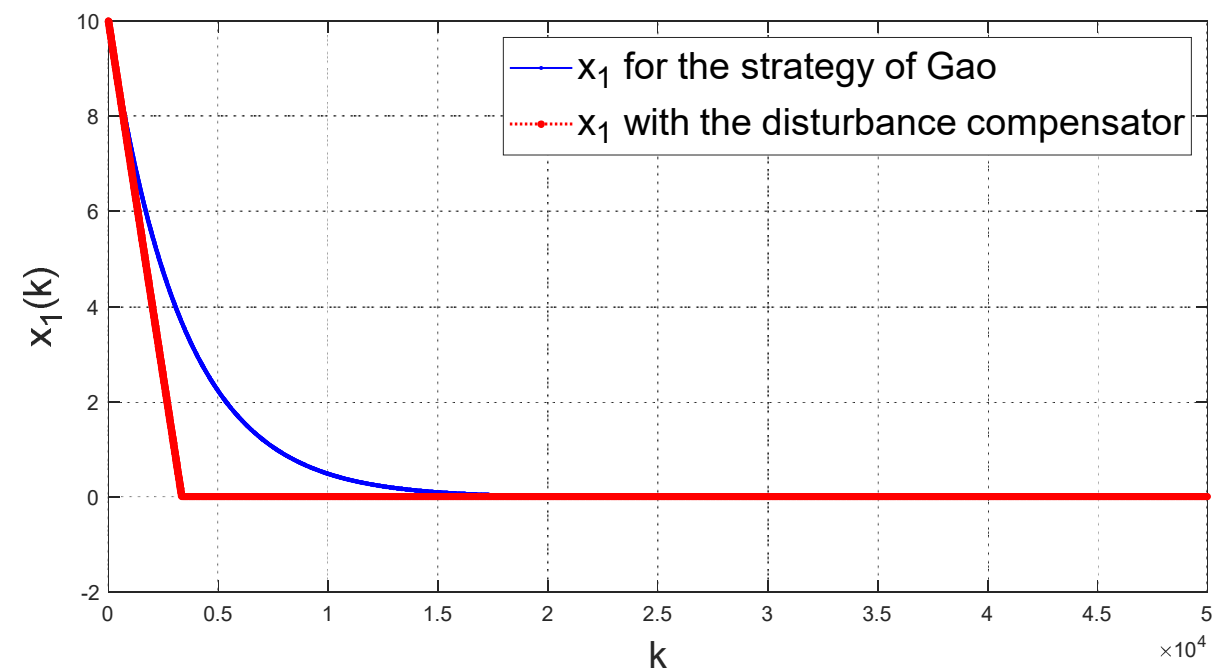

Figure 6. $x_{1}(k)$ for both control strategies.

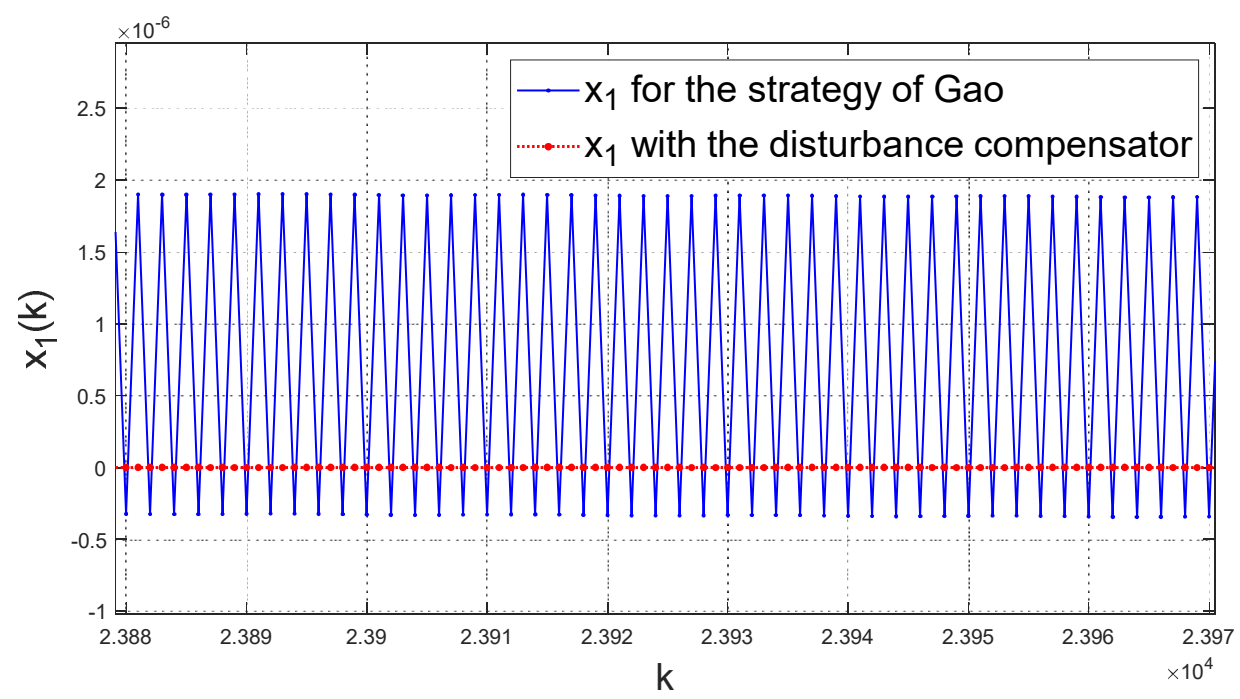

Figure 7. $x_{1}(k)$ for both control strategies in the sliding phase.

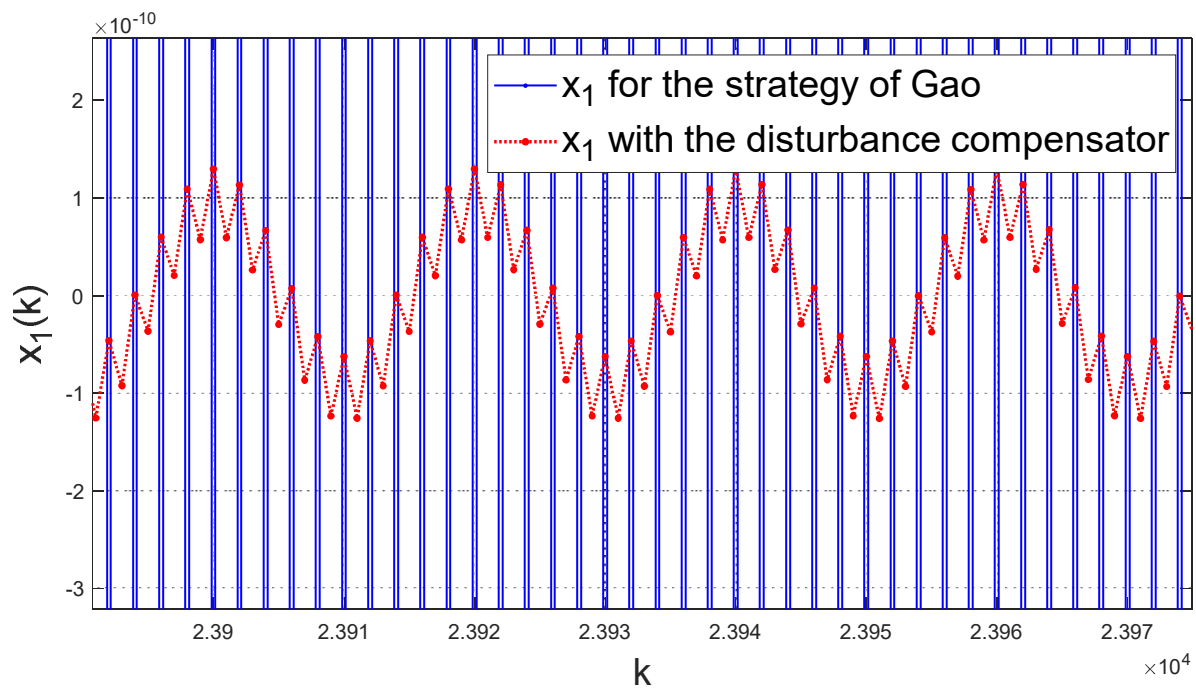

Figure 8. $x_{1}(k)$ for both control strategies in the sliding phase (zoomed). 


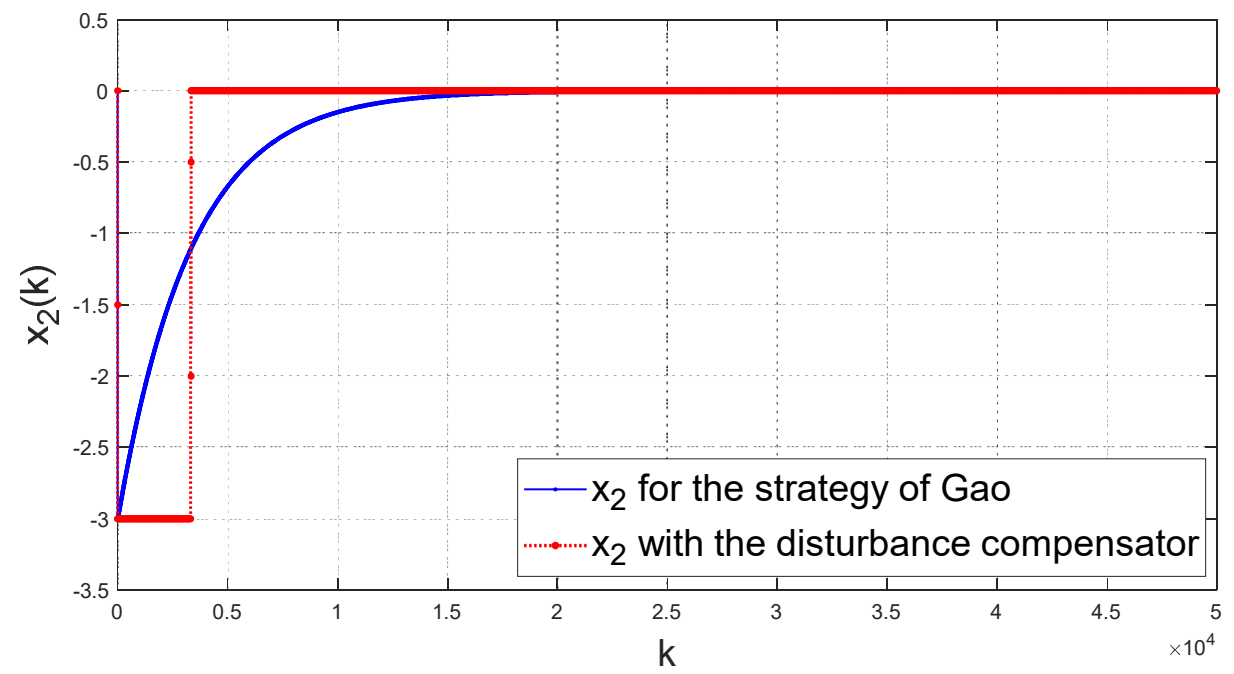

Figure 9. $x_{2}(k)$ for both control strategies.

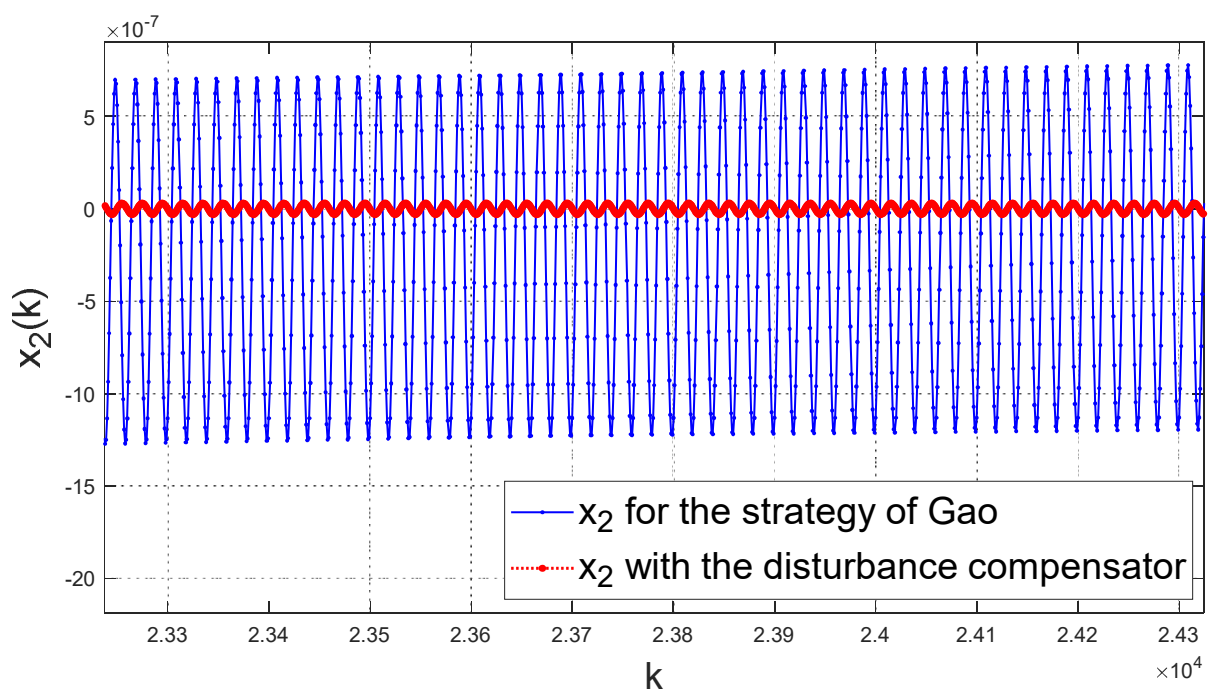

Figure 10. $x_{2}(k)$ for both control strategies in the sliding phase.

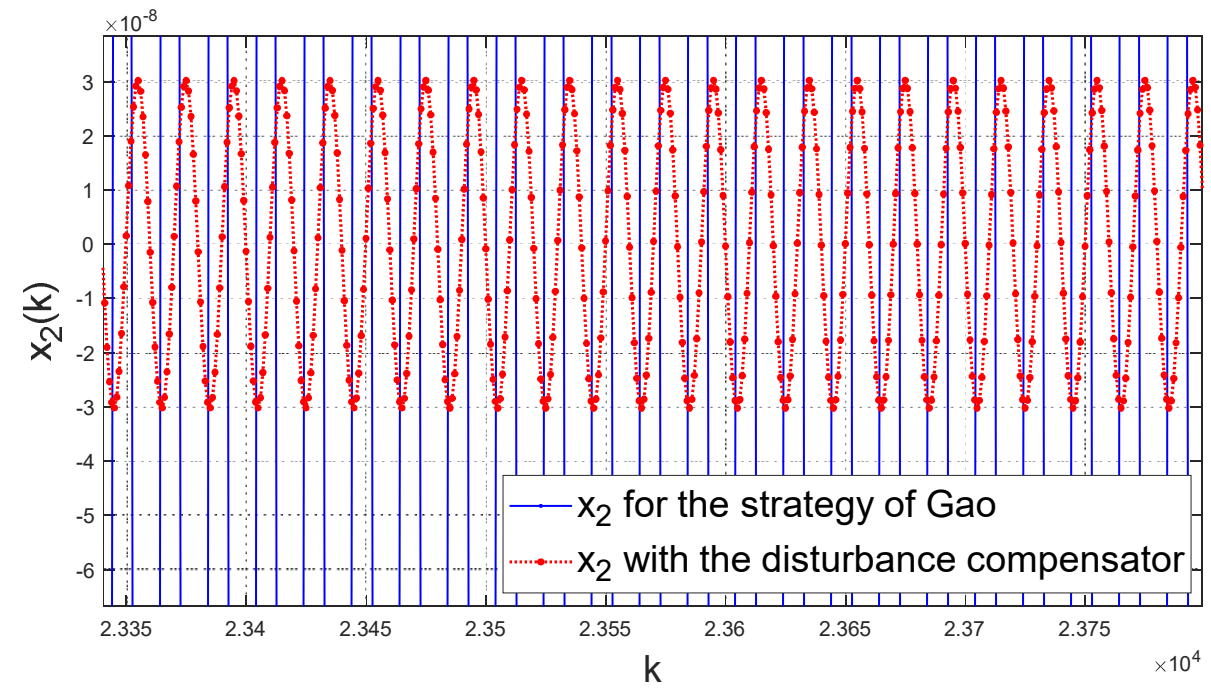

Figure 11. $x_{2}(k)$ for both control strategies in the sliding phase (zoomed). 


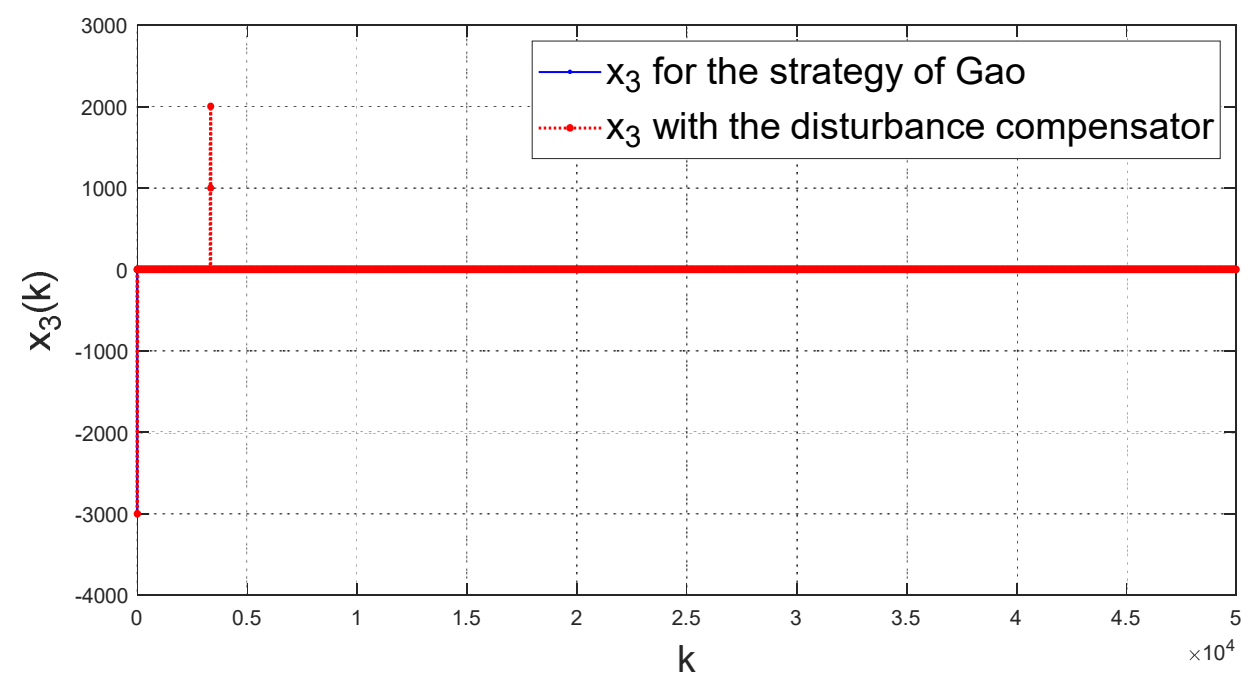

Figure 12. $x_{3}(k)$ for both control strategies.

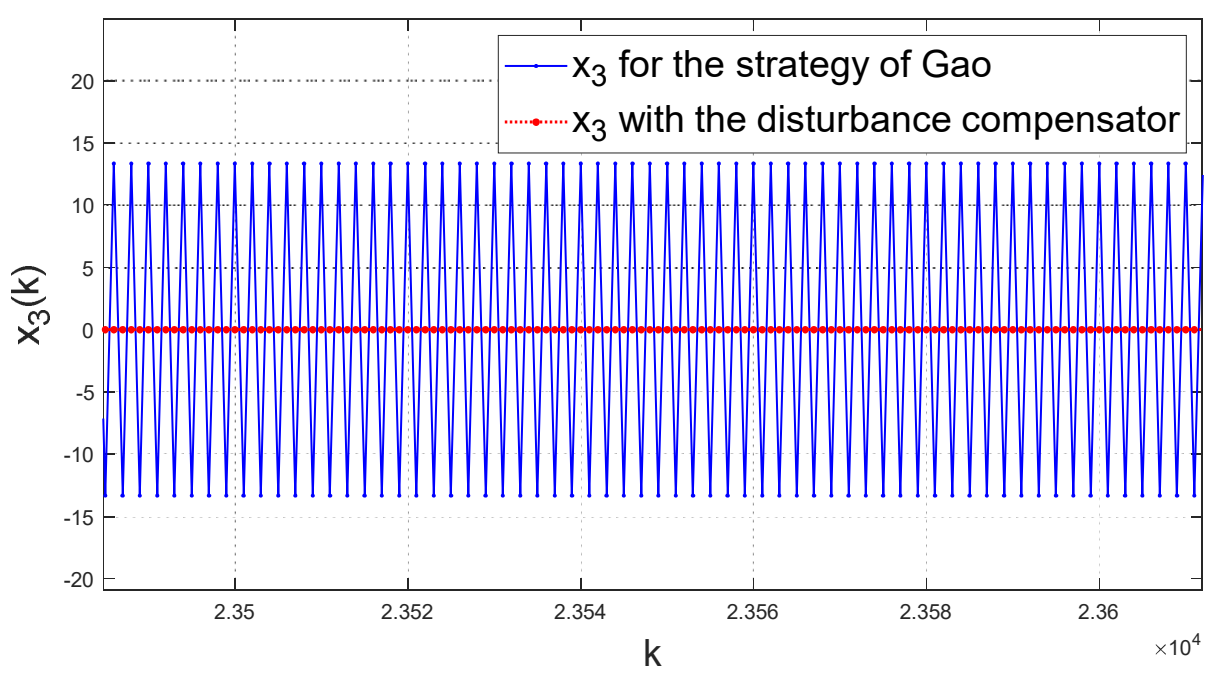

Figure 13. $x_{3}(k)$ for both control strategies in the sliding phase.

Figures 2 and 3 present the evolution of the discrete-time sliding variable $s(k)=c e(k)$. As visible, both sliding variables converge to zero in finite time and the quasi-sliding motion appears. In Gao's case, the quasi-steady state is reached for $t \geq 20.367 \mathrm{~s}$, while with our control strategy the system oscillates around the equilibrium point for any $t \geq 3.334 \mathrm{~s}$. Therefore, our strategy ensured faster convergence to the sliding manifold without an additional control effort. Gao's strategy resulted in the ultimate bandwidth of $2 \times 6.671 \times 10^{-6}$. Applying the trajectory following the strategy with a compensator, we achieved a reduction of this band to $2 \times 1.98 \times 10^{-10}$. Moreover, Figures 4 and 5 clearly show that apart from reducing the ultimate bandwidth and shortening the reaching phase, our new control method satisfies the same control signal limitation as the original strategy and significantly reduces the control effort in the quasi-steady state. Therefore, the closed-loop system with our new control strategy is more energy-saving, which constitutes an important factor in the control design, especially in the case of power systems or electric motor drives.

Finally, Figures 6-14 present the evolution of the state variables $x_{1}(k), x_{2}(k)$, and $x_{3}(k)$ for both control methods. It is clear that the errors of all state variables have been reduced as well. 


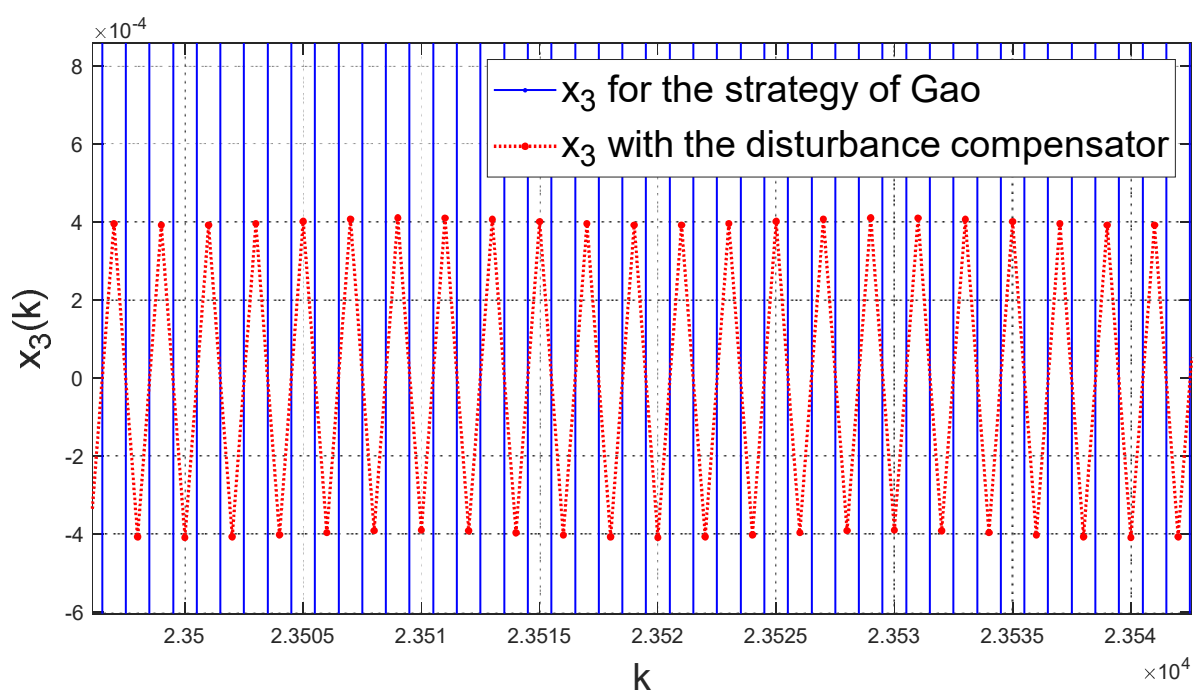

Figure 14. $x_{3}(k)$ for both control strategies in the sliding phase (zoomed).

From the presented graphs we may see the maximum state variables error values. For Gao's method, the errors are:

$$
\begin{gathered}
\left|e_{1}(k)\right| \leq 1.9 \times 10^{-6}, \\
\left|e_{2}(k)\right| \leq 1.3 \times 10^{-6}, \\
\left|e_{3}(k)\right| \leq 13.5 .
\end{gathered}
$$

Our control method reduced the maximum state error values to:

$$
\begin{aligned}
\left|e_{1}(k)\right| & \leq 1.4 \times 10^{-10}, \\
\left|e_{2}(k)\right| & \leq 3.2 \times 10^{-8}, \\
\left|e_{3}(k)\right| & \leq 4.1 \times 10^{-4},
\end{aligned}
$$

which is consistent with Theorem 3. This shows that the introduced control scheme ensures a significant improvement of the robustness of the system along with a reduction of the control effort. Lastly, to precisely show the improvement we define a performance quality index for the discrete-time system as:

$$
P Q I=\sum_{k=0}^{50,000}\left|e_{i}(k)\right|,
$$

where $i=1,2,3$. The obtained index values are presented in Table 1 .

Table 1. Performance Quality Index.

\begin{tabular}{cccc}
\hline \multirow{2}{*}{ Control Strategy } & \multicolumn{3}{c}{ PQI } \\
\cline { 2 - 4 } & $x_{\mathbf{1}}$ & $x_{\mathbf{2}}$ & $x_{\mathbf{3}}$ \\
\hline Gao's control & 32.89 & 10.17 & 401.36 \\
New control method & 16.68 & 10 & 6.02 \\
\hline
\end{tabular}

\section{Conclusions}

This paper proposed a new discrete-time sliding mode control method based on the time-varying sliding surface concept. The control algorithm assumes the application of a time-varying sliding surface, whose shift is obtained according to the reference sliding variable profile. The pre-generated reference profile manifests all the properties required from the actual system, so it converges to zero in finite time and exhibits the zigzagging motion around the equilibrium point. Such a reference profile is obtained with a new- 
reaching law. Next, the sliding mode control law for the actual system is developed so that it drives the representative point to the desired position with accuracy to the single-step disturbance. It is proved that the proposed control method ensures finite time convergence of the representative point of the system to an a priori known band around the designed sliding plane and the width of this band is of $O(T)$ order. Moreover, it provides a restriction of the rate of change of the sliding variable, which results in a reduction of the control effort. As the control magnitudes are limited and chattering effects are reduced, the system becomes more efficient and energy-saving. This achievement may be especially important in practical applications, for example in the control of power electronics systems or electrical motor drives, where reduction of chattering is one of the main issues. Lastly, we supplement our strategy with a disturbance compensator. Its task is to calculate the disturbance value from two previous steps, based on the previous and current state measurement, and use it to compensate for the disturbance in the current step. As the second-order difference of the discretized disturbance is of $O\left(T^{3}\right)$ order, the application of such a compensator ensures that both the sliding variable and all the state variables belong to the band of $O\left(T^{3}\right)$ order. Therefore, the robustness of the system is significantly improved and the energy consumption is reduced. In short, the originality of this paper is manifested in a new efficient reaching law combined with a time-varying sliding surface approach and a disturbance compensator, which results in the restriction of the quasi-sliding mode bandwidth as well as the maximum deviations of all state variables from their demand values to $O\left(T^{3}\right)$ order. As follows, chattering and the control input are minimized, so the system becomes more efficient. Finally, the paper is concluded with a simulation example, which shows that the proposed control strategy not only provides the reduction of the quasi-sliding mode bandwidth but also ensures faster convergence than the conventional method of Gao [27], without increasing the control input.

Author Contributions: Conceptualization, A.B. and K.A.; methodology, A.B. and K.A.; validation, A.B. and K.A.; formal analysis, K.A.; investigation, K.A; writing-original draft preparation, K.A.; writing-review and editing, A.B.; visualization, K.A.; supervision, A.B.; All authors have read and agreed to the published version of the manuscript.

Funding: This research received no external funding.

Institutional Review Board Statement: Not applicable.

Informed Consent Statement: Not applicable.

Data Availability Statement: Not applicable.

Conflicts of Interest: The authors declare no conflict of interest.

\section{References}

1. Emelyanov, S.V. Variable Structure Control Systems; Nauka: Moscow, Russia, 1967.

2. Itkis, U. Control Systems of Variable Structures; Wiley: New York, NY, USA, 1976.

3. Utkin, V.I. Variable structure systems: Present and future. Autom. Remote Control 1984, 44, 1105-1220.

4. Edwards, C. A practical method for the design of sliding mode controllers using linear matrix inequalities. Automatica 2004, 40, 1761-1769. [CrossRef]

5. DeCarlo, R.A.; Żak, S.; Matthews, G. Variable structure control of nonlinear multivariable systems: A tutorial. Proc. IEEE 1988, 76, 212-232. [CrossRef]

6. Slotine, J.E. Sliding controller design for nonlinear system. Int. J. Control 1989, 40, 421-434. [CrossRef]

7. Hung, J.Y.; Gao, W.B.; Hung, J.C. Variable structure control: A survey. IEEE Trans. Ind. Electron. 1993, 40, 2-22. [CrossRef]

8. Zinober, A.S.I. Variable Structure and Lyapunov Control; Springer: London, UK, 1994.

9. Edwards, C.; Colet, E.F.; Fridman, L. Advances in variable structure and sliding mode control. In Lecture Notes in Control and Information Sciences; Springer: Berlin, Germany, 2006; Volume 334.

10. Edwards, C.; Spurgeon, S. Sliding Mode Control: Theory and Applications; Taylor \& Francis: London, UK, 1998.

11. Mobayen, S.; Bayat, F.; Lai, C.-C.; Taheri, A.; Fekih, A. Adaptive global sliding mode controller design for perturbed DC-DC buck converters. Energies 2021, 14, 1249. [CrossRef]

12. Oucheriah, S.; Guo, L. PWM-based adaptive sliding-mode control for boost DC-DC converters. IEEE Trans. Ind. Electron. 2013, 60, 3291-3294. [CrossRef] 
13. Gao, P.; Zhang, G.; Lv, X. Model-free control using improved smoothing extended state observer and super-twisting nonlinear sliding mode control for PMSM drives. Energies 2021, 14, 922. [CrossRef]

14. Tran, A.-T.; Minh, B.L.N.; Huynh, V.V.; Tran, P.T.; Amaefule, E.N.; Phan, V.-D.; Nguyen, T.M. Load frequency regulator in interconnected power system using second-order sliding mode control combined with state estimator. Energies 2021, 14, 863. [CrossRef]

15. Draženović, B. The invariance conditions in variable structure systems. Automatica 1969, 5, 287-295. [CrossRef]

16. Levant, A. Sliding order and sliding accuracy in sliding mode control. Int. J. Control 1993, 58, 1247-1263. [CrossRef]

17. Choi, S.B.; Cheong, C.C.; Park, D.W. Moving switching surfaces for robust control of second-order variable structure systems. Int. J. Control 1993, 58, 229-245. [CrossRef]

18. Choi, S.B.; Park, D.W.; Jayasuriya, S. A time-varying sliding surface for fast and robust tracking control of second-order uncertain systems. Automatica 1994, 30, 899-904. [CrossRef]

19. Betin, F.; Pinchon, D.; Capolino, G. A time-varying sliding surface for robust position control of a DC motor drive. IEEE Trans. Ind. Electron. 2002, 49, 462-473. [CrossRef]

20. Milosavljević, C. General conditions for the existence of a quasi-sliding mode on the switching hyperplane in discrete variable structure systems. Autom. Remote Control 1985, 46, 307-314.

21. Sira-Ramirez, H. Non-linear discrete variable structure systems in quasi-sliding mode. Int. J. Control 1991, 54, 1171-1187. [CrossRef]

22. Sarpturk, S.Z.; Istefanopulos, Y.; Kaynak, O. On the stability of discrete-time sliding mode control systems. IEEE Trans. Autom. Control 1987, 22, 930-932. [CrossRef]

23. Kotta, U.; Sarpturk, S.Z.; Istefanopulos, Y. Comments on 'On the stability of discrete-time sliding mode control systems' [with reply]. IEEE Trans. Autom. Control 1989, 34, 1021-1022. [CrossRef]

24. Utkin, V.I.; Drakunov, S.V. On discrete-time sliding modes. IFAC Proc. Vol. 1989, 22, 273-278.

25. Bartolini, G.; Ferrara, A.; Utkin, V.I. Adaptive sliding mode control in discrete-time systems. Automatica 1995, 31, 769-773. [CrossRef]

26. Furuta, K. Sliding mode control of a discrete system. Syst. Control Lett. 1990, 14, 145-152. [CrossRef]

27. Gao, W.B.; Wang, Y.; Homaifa, A. Discrete-time variable structure control systems. IEEE Trans. Ind. Electron. 1995, 42, 117-122.

28. Veselić, B.; Perunicic-Draženović, B.; Milosavljević, C. Improved discrete-time sliding-mode position control using Euler velocity estimation. IEEE Trans. Ind. Electron. 2010, 57, 3840-3847. [CrossRef]

29. Ma, H.; Wu, J.; Xiong, Z. Discrete-time sliding-mode control with improved quasi-sliding-mode domain. IEEE Trans. Ind. Electron. 2016, 63, 6292-6304. [CrossRef]

30. Chakrabarty, S.; Bandyopadhyay, B. A generalized reaching law for discrete-time sliding mode control. Automatica 2015, 52, 83-86. [CrossRef]

31. Wu-Chung, S.; Drakunov, S.V.; Ozguner, U. An $\mathrm{O}\left(\mathrm{T}^{2}\right)$ boundary layer in sliding mode for sampled-data systems. IEEE Trans. Autom. Control 2000, 45, 482-485. [CrossRef]

32. Rezig, S.; Ezzeddine, W.; Turki, S.; Rezg, N. Mathematical Model for Production Plan Optimization-A Case Study of Discrete Event Systems. Mathematics 2020, 8, 955. [CrossRef]

33. Golo, G.; Milosavljević, C. Robust discrete-time chattering free sliding mode control. Syst. Control Lett. 2000, 41, 19-28. [CrossRef]

34. Du, H.; Yu, X.; Chen, M.Z.Q.; Li, S. Chattering-free discrete-time sliding mode control. Automatica 2016, 68, 87-91. [CrossRef]

35. Shah, D.; Mehta, A. Discrete-Time Sliding Mode Control for Networked Control System. In Studies in Systems, Decision and Control; Springer: Singapore, 2018.

36. Adamiak, K. Reference Sliding Variable Based Chattering-Free Quasi-Sliding Mode Control. IEEE Access 2020, 8, 133086-133094. [CrossRef]

37. Abidi, K.; Xu, J.; Yu, X. On the discrete-time integral sliding-mode control. IEEE Trans. Autom. Control 2007, 52, 709-715. [CrossRef]

38. Qu, S.; Xia, X.; Zhang, J. Dynamics of discrete-time sliding-mode-control uncertain systems with a disturbance compensator. IEEE. Trans. Ind. Electron. 2013, 61, 3502-3510. [CrossRef] 